\title{
Mean-Field Limits of the Quantum Potts Model
}

\author{
A. Cant and Paul A. Pearce \\ Department of Theoretical Physics, Research School of Physical Sciences, The Australian National \\ University, Canberra, A.C.T., 2601, Australia
}

\begin{abstract}
We consider the q-component quantum Potts model on a $d$ dimensional cubic lattice with symmetry breaking and transverse fields. The model is solved exactly in two special limiting cases: 1) the infinite latticedimensionality $(d \rightarrow \infty)$ limit and 2$)$ the limit of infinitely-weak, long-range interactions of Kac type. In each case the resulting free energy and its first partial derivatives (order parameters) are shown to be identical to the corresponding mean-field expressions.
\end{abstract}

\section{Introduction}

The Potts model is a model of central interest in statistical mechanics as is evidenced by the recent and extensive review article by $\mathrm{Wu}$ [1]. Although this model is a simple generalization of the 2-component Ising model to a $q$-component model, it exhibits much richer critical behaviour. Of particular interest is the order of the phase transition as one varies the lattice dimension $d$ and the number of components $q$, regarded as continuous parameters. Mean-field theory [2] predicts a continuous transition for $q \leqq 2$ and a first-order transition for all $q>2$, independent of the lattice dimension $d$. However, Baxter's exact result [3] in two dimensions shows a continuous transition for $q \leqq 4$ and a first-order transition for $q>4$. In general, it is now believed that there exists a critical value $q_{c}(d)$, with a non-trivial dependence on the dimension $d$ (see Fig. 2 in [1]), such that the mean-field prediction is correct for $q>q_{c}(d)$. In addition, renormalizationgroup arguments [4] indicate that the mean-field predictions are correct for $d>4$. It is thus known that $q_{c}(2)=4$ and $q_{c}(d)=2$ for $d>4$. An obvious question is what is the value of $q_{c}(d)$ for $d=3$, in particular, is $q_{c}(3)$ greater than or less than 3 ? For some time the usual series expansions [5] and renormalization-group analyses [6] gave conflicting answers, but the weight of opinion now seems to be that $q_{c}(3)<3$, that is, in three dimensions the 3-component Potts model undergoes a first-order transition.

Recently, a new attack has been made on these problems by looking at the quantum Hamiltonian (field theory) version [7] of the Potts model. Mean-field 
theory of the quantum Potts model [8] again predicts a continuous phase transition for $q \leqq 2$ and a first-order transition for $q>2$, independent of the lattice dimension $d$. Indeed, the initial motivation for studying the quantum model was the underlying belief that $d$-dimensional classical models and their $(d-1)$ dimensional quantum Hamiltonian counterparts have the same phase diagrams and lie in the same universality class [9]. This is certainly true for the $d=2$ Potts case, where Baxter's results [3] can be carried over [10] to the one-dimensional quantum Potts model, and is borne out in higher dimensions by approximate calculations. In particular, by using $1 / q$-expansions for the $(d-1)$-dimensional quantum Potts model, Kogut and co-workers [11] have obtained the remarkable results $q_{c}(3)=2.6 \pm 0.1$ and $q_{c}(d)=2.00 \pm 0.05$ for all $d \geqq 4$. The quantum Potts model has thus clearly emerged as a model worthy of study in its own right.

For many lattice spin systems, it is known [12-15] that mean-field theory becomes exact in certain special limiting cases. Here we shall prove analogous results for a general $q$-component quantum Potts model. This model includes both the classical and the usual (transverse) quantum Potts models as special cases. More specifically, we shall show that the mean-field theory of the general quantum Potts model becomes exact in the following limits: 1) the infinite latticedimensionality $(d \rightarrow \infty)$ limit and 2$)$ the limit of infinitely weak, long-range interactions of Kac type.

We also expect that the mean-field theory becomes exact in the manycomponent $(q \rightarrow \infty)$ limit. This was proved by graphical methods in [15] for the classical Potts model. However, we are not able to prove it for the general quantum model by the methods used here. Although we shall not be concerned with the lattice gauge Potts model here, it is interesting to note that recently this model has been solved exactly [16] in the $q \rightarrow \infty$ limit, yielding mean-field results for the thermodynamic functions.

The rest of this section is devoted to giving a precise statement of our results. For convenience, we describe the classical Potts model before introducing the full quantum model.

The Hamiltonian of the classical Potts model is

$$
H=-\frac{1}{2} \sum_{j, k} J_{j k} \delta\left(\sigma_{j}, \sigma_{k}\right)-\xi \sum_{j} \delta\left(\sigma_{j}, 1\right)
$$

where $\delta(\cdot, \cdot)$ is the Kronecker delta, $\xi \geqq 0$ is an external symmetry-breaking field and the parameters $J_{j k}=J_{k j} \geqq 0$ are pair interaction strengths (with $J_{j j}=0$ ). For simplicity, we shall always take sums on $j$ and $k$ to be over the $N=v^{d}$ lattice vectors in $\mathbb{Z}^{d}$ of a $d$-dimensional cube of side $v$. At each lattice site $j$, the spin $\sigma_{j}$ is restricted to one of $q$ distinct values : in order to make contact with the quantum model, we shall assume that $\sigma_{j}=1, \omega, \omega^{2}, \ldots, \omega^{q-1}$, with $\omega=\exp (2 \pi i / q)$ a $q^{\text {th }}$ root of unity. The partition function for the classical model can then be written as

$$
Z_{N}=\sum_{\sigma_{1}=1}^{\omega^{q-1}} \ldots \sum_{\sigma_{N}=1}^{\omega^{q-1}} \exp (-\beta H),
$$

where $\beta=1 / k_{B} T$ is the inverse temperature and the sums extend over all values of the spins. 
The quantum Potts model is a generalization of the classical Potts model. The Hamiltonian we shall consider is

$$
H=-\frac{1}{2} q^{-1} \sum_{j, k} \sum_{\alpha=1}^{q} J_{j k}\left(\Omega_{j}^{\dagger} \Omega_{k}\right)^{\alpha}-\xi q^{-1} \sum_{j} \sum_{\alpha=1}^{q} \Omega_{j}^{\alpha}-\eta q^{-1} \sum_{j} \sum_{\alpha=1}^{q} M_{j}^{\alpha},
$$

where the lattice structure and interactions are as described previously, with $\eta \geqq 0$ an additional (transverse) field. The spin operators (matrices) $\Omega_{j}$ and $M_{j}$ commute at different sites. At the same site, however, they do not commute but obey the $\mathbb{Z}_{q}$ algebra :

$$
M_{j} \Omega_{j}=\omega \Omega_{j} M_{j}, \quad M_{j}^{\dagger} \Omega_{j}=\omega^{-1} \Omega_{j} M_{j}^{\dagger}, \quad \Omega_{j}^{q}=M_{j}^{q}=I,
$$

where $I$ is the identity and the dagger denotes the Hermitian conjugate. In particular, these operators can be represented as direct products of $N q \times q$ matrices

$$
\begin{gathered}
\Omega_{j}=I \otimes \ldots \otimes I \otimes \Omega \otimes I \otimes \ldots \otimes I, \\
M_{j}=I \otimes \ldots \otimes I \otimes M \otimes I \otimes \ldots \otimes I,
\end{gathered}
$$

where the matrices $\Omega$ and $M$, occuring in the $j^{\text {th }}$ positions, are given by

$$
\Omega=\left[\begin{array}{cccc}
1 & 0 & \ldots & 0 \\
0 & \omega & \ldots & 0 \\
\vdots & & \ddots & \\
0 & 0 & \ldots & \omega^{q-1}
\end{array}\right], \quad M=\left[\begin{array}{cccc}
0 & 1 & 0 & \ldots \\
0 & 0 & 1 & \ldots \\
\vdots & & & \ddots \\
1 & 0 & 0 & \ldots
\end{array}\right] .
$$

We shall work only in this representation, in which the $\Omega_{j}$ are simultaneously diagonal. The partition function is now

$$
Z_{N}=\operatorname{Tr} \exp (-\beta H)
$$

where $H$ is the quantum Hamiltonian and $\operatorname{Tr}$ denotes the matrix trace. The free energy per spin $\psi$ in the thermodynamic limit is given by

$$
\beta \psi(\beta)=-\lim _{N \rightarrow \infty} N^{-1} \ln Z_{N} .
$$

The quantum Potts model reduces to the classical model when the transverse field $\eta$ is set to zero: in this case the Hamiltonian (1.3) is diagonal and the partition function (1.7) reduces to (1.2) after repeated use of the identity

$$
\delta\left(\sigma, \sigma^{\prime}\right)=q^{-1} \sum_{\alpha=1}^{q}\left(\bar{\sigma} \sigma^{\prime}\right)^{\alpha},
$$

where $\sigma$ and $\sigma^{\prime}$ are $q^{\text {th }}$ roots of unity and the bar denotes complex conjugate. The transverse Ising model is also obtained as a special case by setting $q=2$. In this case $\Omega$ and $M$ are familiar Pauli matrices.

In the sequel we shall always assume that the interactions are ferromagnetic and translationally invariant, i.e.

$$
J_{j k}=J_{k j}=J(j-k) \geqq 0,
$$


and impose periodic boundary conditions so that

$$
J_{N}=\sum_{j} J_{j k}
$$

does not depend on $k$. In addition, we shall also assume

$$
J_{\infty}=\lim _{N \rightarrow \infty} J_{N}<\infty,
$$

so that the limiting free energy (1.8) exists [17].

Under these assumptions the result we prove can be stated in two parts as follows :

Theorem. 1) Let $\psi_{d}(\beta)$ be the free energy (1.8) for the quantum Potts model (1.3) with nearest-neighbor interactions given by

$$
J_{j k}= \begin{cases}J / 2 d & |j-k|=1 \\ 0 & \text { otherwise } .\end{cases}
$$

Then

$$
\lim _{d \rightarrow \infty} \psi_{d}(\beta)=\psi_{\mathrm{MF}}(\beta, J),
$$

where $\psi_{\mathrm{MF}}$ is the mean-field free energy given by

$$
\begin{aligned}
\psi_{\mathrm{MF}}(\beta, J)= & \min _{x \in \mathbb{R}}\left\{\frac{1}{2} q^{-1}(q-1) J x^{2}-\frac{1}{2} q^{-1}(q-2) J x-\frac{1}{2}\left(q^{-1} J+\xi+\eta\right)\right. \\
& -\beta^{-1} \ln \left[2 \cosh \left[\frac{1}{2} \beta\left((J x+\xi)^{2}-2 q^{-1}(q-2)(J x+\xi) \eta+\eta^{2}\right)^{1 / 2}\right]\right. \\
& \left.\left.+(q-2) \exp \left[-\frac{1}{2} \beta(J x+\xi+\eta)\right]\right]\right\} .
\end{aligned}
$$

2) Let $\psi_{\gamma}(\beta)$ be the free energy (1.8) for Kac type interactions

$$
J_{j k}=J(j-k)=\gamma^{d} \varrho(\gamma|j-k|), \quad j \neq k,
$$

where it is assumed that $\varrho(r)$ is everywhere bounded and that

$$
J=\lim _{\gamma \rightarrow 0+} \lim _{N \rightarrow \infty} J_{N}=\int_{\mathbb{R}^{d}} \varrho(|\mathbf{r}|) d \mathbf{r}
$$

exists as a Riemann integral. Then

$$
\lim _{\gamma \rightarrow 0+} \psi_{\gamma}(\beta)=\psi_{\mathrm{MF}}(\beta, J) .
$$

An immediate corollary to this theorem is that, in the two limits considered, the order parameters for the quantum Potts model (1.3) are also given by their corresponding mean-field expressions. To see this, we observe that the free energies $\psi_{d}(\beta)$ and $\psi_{\gamma}(\beta)$ are concave functions [17] of the fields $\xi$ and $\eta$; it therefore follows, by a result of Griffiths [18], that taking the limit commutes with the operation of taking the first partial derivative with respect to $\xi$ or $\eta$, so that, for example

$$
\lim _{d \rightarrow \infty} \frac{\partial}{\partial \xi} \psi_{d}(\beta)=\frac{\partial}{\partial \xi} \lim _{d \rightarrow \infty} \psi_{d}(\beta)=\frac{\partial}{\partial \xi} \psi_{\mathrm{MF}}(\beta, J)
$$


We shall not enter here into the details of the order parameters or phase transitions of the general mean-field model given by (1.13). Instead, we refer the reader to the discussions given in [2] for the classical Potts model $(\eta=0)$ and [8] for the transverse Potts model $(\xi=0, J \eta=1)$. It is worth pointing out, however, that for the classical Potts model $(\eta=0)$, the mean-field free energy (1.13) becomes

$$
\psi_{\mathrm{MF}}(\beta, J)=\min _{x \in \mathbb{R}}\left\{\frac{1}{2} q^{-1}(q-1) J x^{2}+q^{-1} J x-\frac{1}{2} q^{-1} J-\beta^{-1} \ln \left[e^{\beta(J x+\xi)}+q-1\right]\right\},
$$

which differs from the expression given by Mittag and Stephen [2] but can easily be shown to be equivalent using the common stationary condition

$$
x=\frac{e^{\beta(J x+\xi)}-1}{e^{\beta(J x+\xi)}+q-1} .
$$

To prove the theorem we shall use the methods of [12] and [14] to obtain upper and lower bounds on the free energy $\psi(\beta)$ which coalesce in the stated limits. In Sect. 2, we use the Bogoliubov variational principle to show that the mean-field free energy always gives an upper bound. In Sect. 3, the lower bound is obtained via a functional integral representation of the Trotter approximation to the partition function.

\section{Upper Bound on the Free Energy}

In this section we shall use Bogoliubov's variational principle [19] to show that

$$
\psi(\beta) \leqq \psi_{\mathrm{MF}}\left(\beta, J_{\infty}\right) .
$$

Before proceeding, however, it is convenient to introduce a vector notation. We define the following $(q-1)$-dimensional vectors:

$$
\begin{aligned}
\boldsymbol{\Omega}_{j} & =\left(\Omega_{j}, \Omega_{j}^{2}, \ldots, \Omega_{j}^{q-1}\right), \\
\mathbf{M}_{j} & =\left(M_{j}, M_{j}^{2}, \ldots, M_{j}^{q-1}\right), \\
\mathbf{1} & =(1,1, \ldots, 1) .
\end{aligned}
$$

Given two $(q-1)$-dimensional vectors $\mathbf{A}$ and $\mathbf{B}$, whose elements are either scalars or $q \times q$ matrices, we define their dot product to be the Hermitian operator

$$
\mathbf{A} \cdot \mathbf{B}=\frac{1}{2} \sum_{\alpha=1}^{q-1}\left(A_{\alpha}^{\dagger} B_{\alpha}+B_{\alpha}^{\dagger} A_{\alpha}\right)
$$

Note that, when $\mathbf{A}, \mathbf{B} \in \mathbb{C}^{q-1}$, the dot product is just the usual real inner product in $\mathbb{C}^{q-1}$ regarded as the vector space $\mathbb{R}^{2(q-1)}$.

Using this compact notation, we can decompose the Hamiltonian (1.3) as

$$
H=H_{0}+H_{1}
$$

where

$$
H_{0}=\frac{1}{2} q^{-1}(q-1) N J_{N} x^{2}-N q^{-1}\left(\frac{1}{2} J_{N}+\xi+\eta\right)-q^{-1}\left(J_{N} x+\xi\right) \sum_{j} \mathbf{1} \cdot \mathbf{\Omega}_{j}-q^{-1} \eta \sum_{j} \mathbf{1} \cdot \mathbf{M}_{j}
$$




$$
H_{1}=-\frac{1}{2} q^{-1} \sum_{j, k} J_{j k}\left(x \mathbf{1}-\mathbf{\Omega}_{j}\right) \cdot\left(x \mathbf{1}-\Omega_{k}\right),
$$

and $x \in \mathbb{R}$ is arbitrary. Bogoliubov's variational principle [19] now states that

$$
\operatorname{Tr} \exp \left[-\beta\left(H_{0}+H_{1}\right)\right] \geqq \exp \left(-\beta\left\langle H_{1}\right\rangle_{0}\right) \operatorname{Tr} \exp \left(-\beta H_{0}\right)
$$

where

$$
\langle\ldots\rangle_{0}=\operatorname{Tr}\left[\ldots \exp \left(-\beta H_{0}\right)\right] / \operatorname{Tr} \exp \left(-\beta H_{0}\right) .
$$

In this case we find

$$
\begin{aligned}
\left\langle H_{1}\right\rangle_{0} & =-\frac{1}{2} q^{-1} \sum_{j, k} J_{j k}\left(x \mathbf{1}-\left\langle\boldsymbol{\Omega}_{j}\right\rangle_{0}\right) \cdot\left(x \mathbf{1}-\left\langle\boldsymbol{\Omega}_{k}\right\rangle_{0}\right) \\
& =-\frac{1}{2} q^{-1}(q-1) \sum_{j, k} J_{j k}\left(x-\left\langle\Omega_{j}\right\rangle_{0}\right)\left(x-\left\langle\Omega_{k}\right\rangle_{0}\right) .
\end{aligned}
$$

Since $J_{j j}=0$, this follows because the expectation $\langle\ldots\rangle_{0}$ factors over the sites and

$$
\left\langle\Omega_{j}\right\rangle_{0}=\left\langle\Omega_{j}^{2}\right\rangle_{0}=\ldots=\left\langle\Omega_{j}^{q-1}\right\rangle_{0} \in \mathbb{R} .
$$

To obtain the desired bound we now choose $x$ to be a solution of

$$
x=\left\langle\Omega_{j}\right\rangle_{0} .
$$

Since $\left\langle\Omega_{j}\right\rangle_{0}$ is independent of $j, x$ will also be independent of $j$. With this choice of $x$, we see that $\left\langle H_{1}\right\rangle_{0}=0$ and hence, from (2.7),

$$
Z_{N} \geqq \operatorname{Tr} \exp \left(-\beta H_{0}\right) \text {. }
$$

From (1.8), (2.5), and (2.11) we therefore conclude that

$$
\begin{aligned}
\psi(\beta) \leqq & \frac{1}{2} J_{\infty} q^{-1}(q-1) x^{2}-q^{-1}\left(\frac{1}{2} J_{\infty}+\xi+\eta\right) \\
& -\beta^{-1} \ln \operatorname{Tr} \exp \left[\beta q^{-1}\left(\left(J_{\infty} x+\xi\right) \mathbf{1} \cdot \mathbf{\Omega}+\eta \mathbf{1} \cdot \mathbf{M}\right)\right],
\end{aligned}
$$

where $J_{\infty}$ is given by (1.10c) and $x$ is any solution of the equation

$$
(q-1) x=\frac{\operatorname{Tr}(\mathbf{1} \cdot \mathbf{\Omega}) \exp \left[\beta q^{-1}\left(\left(J_{\infty} x+\xi\right) \mathbf{1} \cdot \mathbf{\Omega}+\eta \mathbf{1} \cdot \mathbf{M}\right)\right]}{\operatorname{Tr} \exp \left[\beta q^{-1}\left(\left(J_{\infty} x+\xi\right) \mathbf{1} \cdot \mathbf{\Omega}+\eta \mathbf{1} \cdot \mathbf{M}\right)\right]} .
$$

But this equation is precisely the condition for the right side of (2.13) to be stationary with respect to variations in $x$. Thus it follows that (2.1) holds with

$$
\begin{aligned}
\psi_{\mathrm{MF}}(\beta, J)= & \min _{x \in \mathbb{R}}\left\{\frac{1}{2} J q^{-1}(q-1) x^{2}-q^{-1}\left(\frac{1}{2} J+\xi+\eta\right)\right. \\
& \left.-\beta^{-1} \ln \operatorname{Tr} \exp \left[\beta q^{-1}((J x+\xi) \mathbf{1} \cdot \mathbf{\Omega}+\eta \mathbf{1} \cdot \mathbf{M})\right]\right\} .
\end{aligned}
$$

In Appendix A we evaluate the matrix trace which appears in (2.15). Using this result then gives the explicit expression (1.13) for the mean-field free energy $\psi_{\mathrm{MF}}(\beta, J)$.

\section{Lower Bound on the Free Energy}

In this section we derive a lower bound on the free energy (1.8). We begin by writing the Hamiltonian (1.3) in vector notation as 


$$
H=-N q^{-1}\left(\frac{1}{2} J_{N}+\xi+\eta\right)-\frac{1}{2} q^{-1} \sum_{j, k} J_{j k} \mathbf{\Omega}_{j} \cdot \mathbf{\Omega}_{k}-q^{-1} \xi \sum_{j} \mathbf{1} \cdot \mathbf{\Omega}_{j}-q^{-1} \eta \sum_{j} \mathbf{1} \cdot \mathbf{M}_{j}
$$

Let us now regard the interactions $J_{j k}$ as the entries of a matrix $J$. Since $J$ is a cyclic matrix it is readily diagonalized [20]. Defining

$$
S_{j k}=N^{-1 / 2} \prod_{\mu=1}^{d} \exp \left(2 \pi i j_{\mu} k_{\mu} / v\right),
$$

where $N=v^{d}$ and $j_{\mu}$ denotes the components of the lattice vector $j$, we have

$$
S^{-1} J S=\operatorname{diag}\left(\lambda_{j}\right)
$$

where

$$
\lambda_{j}=\lambda\left(\frac{2 \pi j_{1}}{v}, \frac{2 \pi j_{2}}{v}, \ldots, \frac{2 \pi j_{d}}{v}\right)
$$

and

$$
\lambda(\theta)=\lambda\left(\theta_{1}, \theta_{2}, \ldots, \theta_{d}\right)=\sum_{k} J(k) e^{i k \cdot \theta} .
$$

To proceed with the derivation of the lower bound we wish to replace the matrix $J$ with a suitable positive definite matrix $K$. This matrix has to be defined differently for the two limits considered in the theorem. For the $\gamma \rightarrow 0+$ limit [Part 2) of the theorem], it is sufficient to set

$$
K_{j k}=J_{j k}+\gamma^{d} \varrho(0) \delta_{j k}=\gamma^{d} \varrho(\gamma|j-k|),
$$

where $\varrho(0)$ is chosen sufficiently large to make $K$ positive definite. The additional diagonal term of course will not contribute in the limit $\gamma \rightarrow 0+$. For the $d \rightarrow \infty$ limit [Part 1) of the theorem], we define the cyclic matrix $K$ by

$$
K_{j k}=|J|_{j k}+\varepsilon \delta_{j k}, \quad(\varepsilon>0),
$$

where the non-negative definite matrix $|J|$ is given by

$$
|J|=S \operatorname{diag}\left(\left|\lambda_{j}\right|\right) S^{-1} .
$$

Since $\sum_{j, k}\left(K_{j k}-J_{j k}\right) \boldsymbol{\Omega}_{j} \cdot \boldsymbol{\Omega}_{k}$ is a positive definite matrix, it follows immediately from the Peierls theorem [17] that

$$
\begin{aligned}
Z_{N} \leqq & \exp \left[\beta N q^{-1}\left(\frac{1}{2} J_{N}+\xi+\eta\right)\right] \operatorname{Tr} \exp \left[\frac{1}{2} \beta q^{-1} \sum_{j, k} K_{j k} \mathbf{\Omega}_{j} \cdot \mathbf{\Omega}_{k}\right. \\
& \left.+\beta q^{-1} \xi \sum_{j} \mathbf{1} \cdot \mathbf{\Omega}_{j}+\beta q^{-1} \eta \sum_{j} \mathbf{1} \cdot \mathbf{M}_{j}\right] .
\end{aligned}
$$

For Hermitian matrices $A$ and $B$, a straightforward generalization of the Golden-Thompson inequality [21] states that $\operatorname{Tr} e^{A+B} \leqq \operatorname{Tr}\left(e^{A / n} e^{B / n}\right)^{n}$, for all positive integers $n$. By Trotter's formula [22], equality is actually achieved in the limit $n \rightarrow \infty$. Applying this inequality to the right side of (3.9) we obtain

$$
Z_{N} \leqq \exp \left[\beta N q^{-1}\left(\frac{1}{2} J_{N}+\xi+\eta\right)\right] Z_{N, n},
$$


where

$$
Z_{N, n}=\operatorname{Tr}\left[\exp \left(\frac{1}{2} \beta q^{-1} n^{-1} \sum_{j, k} K_{j k} \mathbf{\Omega}_{j} \cdot \mathbf{\Omega}_{k}+\beta q^{-1} n^{-1} \xi \sum_{j} \mathbf{1} \cdot \mathbf{\Omega}_{j}\right) \exp \left(\beta q^{-1} n^{-1} \eta \sum_{j} \mathbf{1} \cdot \mathbf{M}_{j}\right)\right]^{n}
$$

We are now in a position to use the identity

$$
\begin{aligned}
\exp \left(\frac{1}{2} \beta \sum_{j, k} K_{j k} \Omega_{j}^{\dagger} \Omega_{k}\right)= & (\beta / 2 \pi)^{N}(\operatorname{Det} K)^{-1} \int_{\mathbb{R}^{2 N}} \prod_{j} d^{2} z_{j} \\
& \cdot \exp \left[-\frac{1}{2} \beta \sum_{j, k} K_{j k}^{-1} \bar{z}_{j} z_{k}+\frac{1}{2} \beta \sum_{j}\left(\bar{z}_{j} \Omega_{j}+z_{j} \Omega_{j}^{\dagger}\right)\right],
\end{aligned}
$$

where $d^{2} z$ means $d(\operatorname{Re} z) d(\operatorname{Im} z)$. This identity is valid for an arbitrary set of diagonal operators $\Omega_{j}$ and any real symmetric positive definite matrix $K$. Applying it to each such term in the product of $2 n$ ordered exponentials in (3.11) gives

$$
\begin{aligned}
Z_{N, n}= & (\beta / 2 \pi q n)^{N n(q-1)}(\operatorname{Det} K)^{n(1-q)} \int_{\mathbb{R}^{2 N n(q-1)}} \prod_{j} \prod_{t=1}^{n} d^{2} \mathbf{z}_{j t} \\
& \cdot \exp \left[-\frac{1}{2} \beta q^{-1} n^{-1} \sum_{j, k} \sum_{t=1}^{n} K_{j k}^{-1} \mathbf{z}_{j t} \cdot \mathbf{z}_{k t}\right] \\
& \cdot \operatorname{Tr} \prod_{t=1}^{n}\left\{\exp \left[\beta q^{-1} n^{-1} \sum_{j}\left(\mathbf{z}_{j t}+\xi \mathbf{1}\right) \cdot \mathbf{\Omega}_{j}\right] \exp \left[\beta q^{-1} n^{-1} \eta \sum_{j} \mathbf{1} \cdot \mathbf{M}_{j}\right]\right\} .
\end{aligned}
$$

Next we need to estimate the trace in (3.13). This we do in three stages. Firstly, in Appendix B, we show that, for even $n$ and arbitrary $\mathbf{z}_{1}, \mathbf{z}_{2}, \ldots, \mathbf{z}_{n} \in \mathbb{C}^{q-1}$ and $\eta \geqq 0$,

$$
\begin{aligned}
& \left|\operatorname{Tr} \prod_{t=1}^{n}\left[\exp \left(q^{-1} n^{-1} \mathbf{z}_{t} \cdot \mathbf{\Omega}\right) \exp \left(q^{-1} n^{-1} \eta \mathbf{1} \cdot \mathbf{M}\right)\right]\right| \\
& \quad \leqq \prod_{t=1}^{n}\left\{\left[1+4 n^{-1} \exp \left(3\left\|\mathbf{z}_{t}\right\|+\eta\right)\right] \cdot \operatorname{Tr} \exp \left[q^{-1}\left(\mathbf{z}_{t} \cdot \mathbf{\Omega}+\eta \mathbf{1} \cdot \mathbf{M}\right)\right]\right\}^{1 / n}
\end{aligned}
$$

Secondly, in Appendix $\mathrm{C}$, we show that, for $\mathbf{z} \in \mathbb{C}^{q-1}$ and $\eta \geqq 0$,

$$
\operatorname{Tr} \exp (\mathbf{z} \cdot \mathbf{\Omega}+\eta \mathbf{1} \cdot \mathbf{M}) \leqq \operatorname{Tr} \exp \left[(q-1)^{-1 / 2}\|\mathbf{z}\| \mathbf{1} \cdot \mathbf{\Omega}+\eta \mathbf{1} \cdot \mathbf{M}\right] \text {. }
$$

Lastly, in Appendix D, we prove the monotonicity property

$$
\frac{\partial}{\partial x} \operatorname{Tr} \exp (x \mathbf{1} \cdot \mathbf{\Omega}+\eta \mathbf{1} \cdot \mathbf{M}) \geqq 0
$$

for $x \geqq 0$ and $\eta \geqq 0$. Combining these inequalities with the triangle inequality $\|\mathbf{z}+\xi \mathbf{1}\| \leqq\|\mathbf{z}\|+(q-1)^{1 / 2} \xi \leqq\|\mathbf{z}\|+q \xi$, we obtain

$$
\begin{aligned}
\mid \operatorname{Tr} & \prod_{t=1}^{n}\left\{\exp \left[\beta q^{-1} n^{-1}\left(\mathbf{z}_{t}+\xi \mathbf{1}\right) \cdot \mathbf{\Omega}\right] \exp \left[\beta q^{-1} n^{-1} \eta \mathbf{1} \cdot \mathbf{M}\right]\right\} \mid \\
\leqq & \prod_{t=1}^{n}\left\{\left[1+4 n^{-1} \exp \left(3 \beta\left\|\mathbf{z}_{t}\right\|+3 \beta q \xi+\beta \eta\right)\right]\right. \\
& \left.\cdot \operatorname{Tr} \exp \left[\beta q^{-1}\left((q-1)^{-1 / 2}\left\|\mathbf{z}_{t}\right\|+\xi\right) \mathbf{1} \cdot \mathbf{\Omega}+\beta q^{-1} \eta \mathbf{1} \cdot \mathbf{M}\right]\right\}^{1 / n}
\end{aligned}
$$


If we now factor out the direct product over $j$ appearing in (3.13) and apply the inequality (3.17) at each site we find

$$
\begin{aligned}
Z_{N, n} \leqq & (\beta / 2 \pi q n)^{N n(q-1)}(\operatorname{Det} K)^{-n(q-1)} \int_{\mathbb{R}^{2 N n(q-1)}} \prod_{j} \prod_{t=1}^{n} d^{2} \mathbf{z}_{j t} \\
& \cdot \exp \left[-\frac{1}{2} \beta q^{-1} n^{-1} \sum_{j, k} \sum_{t=1}^{n}\left(K_{j k}^{-1}-\zeta^{-1} \delta_{j k}\right) \mathbf{z}_{j t} \cdot \mathbf{z}_{k t}\right] \\
& \cdot \prod_{j} \prod_{t=1}^{n} \exp \left\{-\frac{1}{2} \beta q^{-1} n^{-1} \zeta^{-1}\left\|\mathbf{z}_{j t}\right\|^{2}\right. \\
& +n^{-1} \ln \operatorname{Tr} \exp \left[\beta q^{-1}\left((q-1)^{-1 / 2}\left\|\mathbf{z}_{j t}\right\|+\xi\right) \mathbf{1} \cdot \mathbf{\Omega}+\beta q^{-1} \eta \mathbf{1} \cdot \mathbf{M}\right] \\
& \left.+n^{-1} \ln \left[1+4 n^{-1} \exp \left(3 \beta\left\|\mathbf{z}_{j t}\right\|+3 \beta q \xi+\beta \eta\right)\right]\right\},
\end{aligned}
$$

where, in anticipation of the next step, we have added and subtracted a term in the exponent with $\zeta>0$ arbitrary.

To obtain the required upper bound on $Z_{N, n}$, our strategy now is to replace each term in the product over $j, t$ in (3.18) by the common maximum. This maximum occurs for $\left\|\mathbf{z}_{j t}\right\|=\|\mathbf{z}\|$ satisfying the stationary condition

$$
\zeta^{-1}\|\mathbf{z}\|=F(\|\mathbf{z}\|)
$$

where

$$
\begin{aligned}
F(x)= & \frac{\operatorname{Tr}(\mathbf{1} \cdot \mathbf{\Omega}) \exp \left[\beta q^{-1}\left((q-1)^{-1 / 2} x+\xi\right) \mathbf{1} \cdot \mathbf{\Omega}+\beta q^{-1} \eta \mathbf{1} \cdot \mathbf{M}\right]}{(q-1)^{1 / 2} \operatorname{Tr} \exp \left[\beta q^{-1}\left((q-1)^{-1 / 2} x+\xi\right) \mathbf{1} \cdot \mathbf{\Omega}+\beta q^{-1} \eta \mathbf{1} \cdot \mathbf{M}\right]} \\
& +\frac{12 q n^{-1} \exp (3 \beta x+3 \beta q \xi+\beta \eta)}{1+4 n^{-1} \exp (3 \beta x+3 \beta q \xi+\beta \eta)}
\end{aligned}
$$

After the maximization of the product over $j, t$ in (3.18), we perform the remaining Gaussian integrals. Setting

$$
x=(q-1)^{-1 / 2 \zeta^{-1}}\|\mathbf{z}\|
$$

we obtain

$$
\begin{aligned}
Z_{N, n} \leqq & \operatorname{Det}\left(I-\zeta^{-1} K\right)^{-n(q-1)} \\
& \cdot \max _{x \in \mathbb{R}}\left\{\exp \left[-\frac{1}{2} \beta q^{-1}(q-1) \zeta x^{2}\right] \cdot \operatorname{Tr} \exp \left[\beta q^{-1}(\zeta x+\xi) \mathbf{1} \cdot \mathbf{\Omega}+\beta q^{-1} \eta \mathbf{1} \cdot \mathbf{M}\right]\right. \\
& \left.\cdot\left[1+4 n^{-1} \exp \left(3 \beta(q-1)^{1 / 2} \zeta x+3 \beta q \xi+\beta \eta\right)\right]\right\}^{N} .
\end{aligned}
$$

The manipulation leading to (3.22) clearly requires the matrix $I-\zeta^{-1} K$ to be positive definite. This will certainly be true if we choose $\zeta$ to be greater than the maximum eigenvalue of $K$, i.e., either

$$
\zeta>J_{N}+\varepsilon \text { or } \zeta>J_{\infty}+\gamma^{d} \varrho(0)
$$

as appropriate. We now take the thermodynamic limit $N \rightarrow \infty$. From (3.9), (3.10), and (3.22), it then follows that 


$$
\begin{aligned}
\psi(\beta)= & -\lim _{N \rightarrow \infty}(\beta N)^{-1} \ln Z_{N} \\
\geqq & \min _{x \in \mathbb{R}}\left\{\frac{1}{2} q^{-1}(q-1) \zeta x^{2}-q^{-1}\left(\frac{1}{2} J_{\infty}+\xi+\eta\right)\right. \\
& -\beta^{-1} \ln \operatorname{Tr} \exp \left[\beta q^{-1}(\zeta x+\xi) \mathbf{1} \cdot \mathbf{\Omega}+\beta q^{-1} \eta \mathbf{1} \cdot \mathbf{M}\right] \\
& \left.-\beta^{-1} \ln \left[1+4 n^{-1} \exp \left(3 \beta(q-1)^{1 / 2} \zeta x+3 \beta q \xi+\beta \eta\right)\right]\right\}+n(q-1) R(\beta, \zeta),
\end{aligned}
$$

where, by Szegö's theorem [23], the remainder term $R(\beta, \zeta)$ is

$$
R(\beta, \zeta)=\beta^{-1} \lim _{N \rightarrow \infty} N^{-1} \ln \operatorname{Det}\left(I-\zeta^{-1}|J|\right)=\beta^{-1}(2 \pi)^{-d} \int_{0}^{2 \pi} \ldots \int d \theta \ln \left(1-\zeta^{-1}|\lambda(\theta)|\right) .
$$

Here $\lambda(\boldsymbol{\theta})$ is given by (3.5) with either $J(0)=0$ or $J(0)=\gamma^{d} \varrho(0)$ as appropriate.

In each of the two limits $d \rightarrow \infty, \gamma \rightarrow 0+$ the remainder term (3.25) vanishes. For the $d \rightarrow \infty$ limit, this was proved in [12]. For the long-range $\gamma \rightarrow 0+$ limit it has been proved in [13]. Evaluating the trace in (3.24) (Appendix A) and taking the limits $n \rightarrow \infty, \zeta \rightarrow J+[$ see (3.23)] after all other limits, we conclude that

$$
\lim _{d \rightarrow \infty} \psi_{d}(\beta) \geqq \psi_{\mathrm{MF}}(\beta, J),
$$

and

$$
\lim _{\gamma \rightarrow 0+} \psi_{\gamma}(\beta) \geqq \psi_{\mathrm{MF}}(\beta, J),
$$

where $\psi_{\mathrm{MF}}(\beta, J)$ is the mean-field free energy given by (1.13). These inequalities, along with the reverse inequality (2.1), prove the theorem stated in Sect. 1.

\section{Appendix A}

In this appendix we prove the following:

Lemma. For arbitrary $x$ and $\eta$,

$$
\begin{aligned}
\operatorname{Tr} \exp (x \mathbf{1} \cdot \mathbf{\Omega}+\eta \mathbf{1} \cdot \mathbf{M})= & \exp \left[\frac{1}{2}(q-2)(x+\eta)\right] \\
& \cdot\left\{2 \cosh \left[\frac{1}{2}\left(q^{2} x^{2}-2 q(q-2) x \eta+q^{2} \eta^{2}\right)^{1 / 2}\right]\right. \\
& \left.+(q-2) \exp \left[-\frac{1}{2} q(x+\eta)\right]\right\} .
\end{aligned}
$$

Proof. We wish to find the eigenvalues of the $q \times q$ matrix

$$
x \mathbf{1} \cdot \mathbf{\Omega}+\eta \mathbf{1} \cdot \mathbf{M}=\left[\begin{array}{ccccc}
(q-1) x & \eta & \ldots & \eta & \eta \\
\eta & -x & \ldots & \eta & \eta \\
\vdots & & \ddots & \vdots & \vdots \\
\eta & \eta & \ldots & -x & \eta \\
\eta & \eta & \ldots & \eta & -x
\end{array}\right] .
$$

Such a matrix has been studied in [8]: it has two eigenvectors of the form $(a, 1,1, \ldots, 1)$ with eigenvalues

$$
\lambda_{1,2}=\frac{1}{2}(q-2)(x+\eta) \pm \frac{1}{2}\left[q^{2} x^{2}-2 q(q-2) x \eta+q^{2} \eta^{2}\right]^{1 / 2},
$$


and $(q-2)$ eigenvectors of the form $\left(0,1, w, w^{2}, \ldots, w^{q-2}\right)$, where $w \neq 1$ is a $(q-1)^{\text {th }}$ root of unity, with degenerate eigenvalues

$$
\lambda_{3}=\lambda_{4}=\ldots=\lambda_{q}=-(x+\eta)
$$

Hence

$$
\operatorname{Tr} \exp (x \mathbf{1} \cdot \mathbf{\Omega}+\eta \mathbf{1} \cdot \mathbf{M})=e^{\lambda_{1}}+e^{\lambda_{2}}+(q-2) e^{\lambda_{3}}
$$

from which (A.1) follows.

\section{Appendix B}

Our aim here is to prove the inequality (3.14). To do this we first prove the following:

Lemma. If $A$ and $B$ are $q \times q$ matrices and $n$ a positive integer, then

$$
\left|\operatorname{Tr}\left[\exp \left(n^{-1} A\right) \exp \left(n^{-1} B\right)\right]^{n}\right| \leqq\left\{1+2 n^{-1} \exp [3(\|A\|+\|B\|)]\right\}|\operatorname{Tr} \exp (A+B)|
$$

where the norm $\|A\|$ is given by

$$
\|A\|=\sup _{\|\mathbf{v}\|=1}\|A \mathbf{v}\|
$$

with $\|\mathbf{v}\|^{2}=\mathbf{v} \cdot \mathbf{v}$ for $\mathbf{v} \in \mathbb{C}^{q}$.

Proof. Let us set

$$
\begin{gathered}
T=\exp \left(n^{-1} A\right) \exp \left(n^{-1} B\right), \\
S=\exp \left[n^{-1}(A+B)\right]
\end{gathered}
$$

then

$$
\left|\frac{\operatorname{Tr}\left(T^{n}\right)}{\operatorname{Tr}\left(S^{n}\right)}\right|=\left|\frac{\operatorname{Tr}\left(T^{n} S^{-n} S^{n}\right)}{\operatorname{Tr}\left(S^{n}\right)}\right| \leqq \varrho\left(T^{n} S^{-n}\right) \leqq\left\|T^{n} S^{-n}\right\|,
$$

where $\varrho(A)$ denotes the spectral radius of $A$. Using the elementary properties of the norm (B.2), i.e.

$$
\|A+B\| \leqq\|A\|+\|B\|, \quad\|A B\| \leqq\|A\|\|B\|, \quad\|I\|=1,
$$

we further estimate that

$$
\left\|T^{n} S^{-n}\right\|=\left\|I+\left(T^{n}-S^{n}\right) S^{-n}\right\| \leqq 1+\left\|T^{n}-S^{n}\right\|\left\|S^{-n}\right\| .
$$

The required result (B.1) thus follows from the estimates (see, for example, Reed and Simon [22, p. 295]):

$$
\begin{gathered}
\left\|S^{-n}\right\| \leqq \exp (\|A\|+\|B\|), \\
\left\|S^{n}-T^{n}\right\| \leqq 2 n^{-1} \exp [2(\|A\|+\|B\|)] .
\end{gathered}
$$

To obtain the inequality (3.14), we can now use the Hölder inequality [24]

$$
\left|\operatorname{Tr} \prod_{t=1}^{n} T_{t}\right| \leqq \prod_{t=1}^{n}\left[\operatorname{Tr}\left(T_{t}^{\dagger} T_{t}\right)^{n / 2}\right]^{1 / n}
$$


with

$$
\begin{gathered}
T_{t}=\exp \left(n^{-1} A_{t}\right) \exp \left(n^{-1} B_{t}\right), \\
A_{t}=q^{-1} \mathbf{z}_{t} \cdot \mathbf{\Omega}, \quad B_{t}=q^{-1} \eta \mathbf{1} \cdot \mathbf{M} .
\end{gathered}
$$

Since $A_{t}$ and $B_{t}$ are Hermitian we find

$$
\begin{aligned}
\operatorname{Tr}\left(T_{t}^{\dagger} T_{t}\right)^{n / 2} & =\operatorname{Tr}\left[\exp \left(n^{-1} B_{t}\right) \exp \left(2 n^{-1} A_{t}\right) \exp \left(n^{-1} B_{t}\right)\right]^{n / 2} \\
& =\operatorname{Tr}\left[\exp \left(2 n^{-1} A_{t}\right) \exp \left(2 n^{-1} B_{t}\right)\right]^{n / 2} .
\end{aligned}
$$

In this last step we have assumed that $n$ is even and used the cyclic property of the trace. Applying the preceding lemma to (B.10) now gives

$$
\begin{aligned}
& \left|\operatorname{Tr} \prod_{t=1}^{n} \exp \left(n^{-1} A_{t}\right) \exp \left(n^{-1} B_{t}\right)\right| \\
& \quad \leqq \prod_{t=1}^{n}\left\{\left[1+4 n^{-1} \exp \left(3\left(\left\|A_{t}\right\|+\left\|B_{t}\right\|\right)\right)\right] \operatorname{Tr} \exp \left(A_{t}+B_{t}\right)\right\}^{1 / n} .
\end{aligned}
$$

Recalling the definitions (B.12) of $A_{t}$ and $B_{t}$, we see that (B.14) gives the desired inequality (3.14), once $\left\|A_{t}\right\|$ and $\left\|B_{t}\right\|$ have been replaced by the simple estimates:

$$
\begin{gathered}
\left\|A_{t}\right\| \leqq q^{-1} \sum_{\alpha=1}^{q-1}\left|z_{t \alpha}\right|\|\Omega\|^{\alpha}=q^{-1} \sum_{\alpha=1}^{q-1}\left|z_{t \alpha}\right| \leqq\left\|\mathbf{z}_{t}\right\|, \\
\left\|B_{t}\right\| \leqq q^{-1} \eta \sum_{\alpha=1}^{q-1}\|M\|^{\alpha}=q^{-1}(q-1) \eta \leqq \eta
\end{gathered}
$$

\section{Appendix C}

In this appendix we prove the inequality (3.15). This inequality follows immediately from the following stronger result:

Lemma. Let $A, B, C$ be the $q \times q$ Hermitian matrices given $b y$

$$
\begin{gathered}
A=\mathbf{z} \cdot \mathbf{\Omega}+(q-1)^{-1 / 2}\|\mathbf{z}\| I, \\
B=(q-1)^{-1 / 2}\|\mathbf{z}\|(\mathbf{1} \cdot \mathbf{\Omega}+I), \\
C=\eta(\mathbf{1} \cdot \mathbf{M}+I) .
\end{gathered}
$$

Then, for any positive integer $n$,

$$
\operatorname{Tr}(A+C)^{n} \leqq \operatorname{Tr}(B+C)^{n} .
$$

Proof. We first show that

$$
\operatorname{Tr} A^{n} \leqq \operatorname{Tr} B^{n} .
$$

For $n=1$, equality holds, i.e. $\operatorname{Tr} A=\operatorname{Tr} B$, because

$$
\operatorname{Tr} \Omega=\mathbf{0} .
$$


For $n=2$, a straightforward calculation gives

$$
\begin{aligned}
\operatorname{Tr} A^{2} & =(q / 4) \sum_{\alpha=1}^{q-1}\left|z_{\alpha}+\bar{z}_{q-\alpha}\right|^{2}+q(q-1)^{-1}\|\mathbf{z}\|^{2} \\
& \leqq q^{2}(q-1)^{-1}\|\mathbf{z}\|^{2}=\operatorname{Tr} B^{2},
\end{aligned}
$$

where the last equality is easily obtained by noting that

$$
\mathbf{1} \mathbf{\Omega}+I=\operatorname{diag}(q, 0,0, \ldots, 0) .
$$

For $n \geqq 3$, we have

$$
\operatorname{Tr} A^{n} \leqq\left(\operatorname{Tr} A^{2}\right)^{n / 2} \leqq\left[q(q-1)^{-1 / 2}\|\mathbf{z}\|\right]^{n}=\operatorname{Tr} B^{n} .
$$

Here the first inequality is a generalization of a standard inequality for $l^{p}$ norms and the second inequality follows from (C.7).

To prove (C.4) we now observe that the entries of the matrix $C$ are all equal to $\eta$. Because it is of this special form, the matrix $C$ can be eliminated from the trace of any product, formed with a diagonal $q \times q$ matrix $D$, by using the cyclic property of the trace and the following identities:

$$
C^{2}=q \eta C, \quad C D C=\eta(\operatorname{Tr} D) C, \quad \operatorname{Tr} C D=\eta \operatorname{Tr} D .
$$

Using (C.7) it follows, for example, that

$$
\operatorname{Tr} A C A^{2} C^{2}=q \eta^{3} \operatorname{Tr} A \cdot \operatorname{Tr} A^{2} \leqq q \eta^{3} \operatorname{Tr} B \cdot \operatorname{Tr} B^{2}=\operatorname{Tr} B C B^{2} C^{2} .
$$

Since this argument holds for any such product, (C.4) can be obtained by using the binomial expansion for non-commuting operators and comparing the traces termby-term. Likewise, by expanding the exponentials, we can prove

$$
\operatorname{Tr} e^{A+C} \leqq \operatorname{Tr} e^{B+C}
$$

This inequality is equivalent to (3.15).

\section{Appendix D}

In this appendix we prove the following:

Lemma. Suppose $x \geqq 0$ and $\eta \geqq 0$. Then

$$
\frac{\partial}{\partial x} \operatorname{Tr} \exp (x \mathbf{1} \cdot \mathbf{\Omega}+\eta \mathbf{1} \cdot \mathbf{M})=\operatorname{Tr}(\mathbf{1} \cdot \mathbf{\Omega}) \exp (x \mathbf{1} \cdot \mathbf{\Omega}+\eta \mathbf{1} \cdot \mathbf{M}) \geqq 0,
$$

Proof. Let $C$ and $D$ be the $q \times q$ Hermitian matrices given by

$$
\begin{gathered}
C=\eta(\mathbf{1} \cdot \mathbf{M}+I), \\
D=\mathbf{1} \cdot \mathbf{\Omega}=\operatorname{diag}(q-1,-1,-1, \ldots,-1) .
\end{gathered}
$$

Then to prove (D.1), i.e.

$$
\operatorname{Tr} D \exp (C+x D) \geqq 0
$$


we expand the exponential, take the trace term-by-term using (C.10), and use the inequalities

$$
\operatorname{Tr} D^{n}=(q-1)^{n} \pm(q-1) \geqq 0 .
$$

\section{References}

1. Wu, F.Y.: The Potts model. Rev. Mod. Phys. 54, 235-268 (1982)

2. Kihara, T., Midzuno, Y., Shizume, J. : Statistics of two-dimensional lattices with many components. J. Phys. Soc. Jpn. 9, 681-687 (1954)

Straley, J.P., Fisher, M.E.: Three-state Potts model and anomalous tricritical points. J. Phys. A 6, 1310-1326 (1973)

Mittag, L., Stephen, M.J.: Mean-field theory of the many component Potts model. J. Phys. A 7, L109-112 (1974)

3. Baxter, R.J.: Potts model at the critical temperature. J. Phys. C 6, L445-448 (1973)

4. Aharony, A., Pytte, E.: First- and second-order transitions in the Potts model near four dimensions. Phys. Rev. B 23, 362-367 (1981)

5. Ditzian, R.V., Oitmaa, J. : Tricritical behaviour in an Ising system and the Potts model. J. Phys. A 7, L61-64 (1974)

Straley, J.P.: Three dimensional Potts model. J. Phys. A 7, 2173-2180 (1974)

Enting, I.G. : Series expansions for the Potts model: high-field expansions. J. Phys. A 7, 1617-1626 (1974)

Kim, D., Joseph, R.I.: High temperature series study of the $q$ component Potts model in two and three dimensions. J. Phys. A 8, 891-904 (1975)

Miyashita, S., Betts, D.D., Elliott, C.J.: High-field series expansions and critical properties for the three-state Potts model. J. Phys. A 12, 1605-1622 (1979)

6. Golner, G.R. : Investigation of the Potts model using renormalization group techniques. Phys. Rev. B 8, 3419-3422 (1973)

Rudnik, J. : $\varepsilon$ expansion for the free energy of the continuous three-state Potts model : evidence for a first-order phase transition. J. Phys. A 8, 1125-1129 (1975)

Zia, R.K.P., Wallace, D.J. : Critical behaviour of the continuous $n$-component Potts model. J. Phys. A 8, 1495-1507 (1975)

Burkhardt, T.W., Knops, H.J.F., den Nijs, M.: Renormalization-group results for the three-state Potts model. J. Phys. A 9, L179-181 (1976)

Southern, B.W. : Kadanoff renormalization for the $s$-state Potts model in three dimensions. J. Phys. A 10, L253-255 (1977)

Nienhuis, B., Riedel, E.K., Schick, M. : q-state Potts model in general dimension. Phys. Rev. B 23, 6055-6060 (1981)

7. Fradkin, E., Susskind, L.: Order and disorder in gauge systems and magnets. Phys. Rev. D 17, 2637-2658 (1978)

Solyóm, J., Pfeuty, P.: Renormalization-group study of the Hamiltonian version of the Potts model. Phys. Rev. B 24, 218-229 (1981)

8. Goldschmidt, Y.Y., Shigemitsu, J.: Quantum Potts gauge-matter systems at finite temperature. Nucl. Phys. B 200 [FS4], 149-210 (1982)

Masperi, L., Omero, C.: Variational approach for the $N$-state spin and gauge Potts model. Nucl. Phys. B 200 [FS4], 121-134 (1982)

9. Kogut, J.B.: An introduction to lattice gauge theory and spin systems. Rev. Mod. Phys. 51, 659-713 (1979)

10. Hamer, C.J.: $Q$-state Potts models in Hamiltonian field theory for $Q \geqq 4$ in $(1+1)$-dimensions. J. Phys. A 14, 2981-3003 (1981)

11. Kogut, J.B., Sinclair, D.K.: $1 / Q$ expansions and the first-order phase transition of the three-state Potts model in three-dimensions. Phys. Lett 81 A, 149-152 (1981); 1/ $Q$ expansions for Potts models in all dimensions. Phys. Lett. 86 A, 38-42 (1981)

Kogut, J.B., Pearson, R.B., Shigemitsu, J.: Hamiltonian Potts model. Institute for Theoretical Physics (University of California) (preprint) (1982) 
12. Pearce, P.A., Thompson, C.J.: The high density limit for lattice spin models. Commun. Math. Phys. 58, 131-138 (1978)

13. Thompson, C.J., Silver, H. : The classical limit of $n$-vector spin models. Commun. Math. Phys. 33, 53-60 (1973)

14. Pearce, P.A., Thompson, C.J.: The anisotropic Heisenberg model in the long-range interaction limit. Commun. Math. Phys. 41, 191-201 (1975)

15. Pearce, P.A., Griffiths, R.B.: Potts model in the many-component limit. J. Phys. A 13, 2143-2148 (1980)

16. Kotecký, R.: Mean-field approximation is exact in the many-component limit of Potts lattice gauge model. Commun. Math. Phys. 82, 391-397 (1981)

17. Ruelle, D.: Statistical mechanics. New York: Benjamin 1969

18. Griffiths, R.B.: A proof that the free energy of a spin system is extensive. J. Math. Phys. 5, 1215-1222 (1964)

19. Girardeau, M.: Variational method for the quantum statistics of interacting particles. J. Math. Phys. 3, 131-139 (1962)

Huber. A.: Methods and problems of theoretical physics. Bowcock. J.E. (ed.). Amsterdam: NorthHolland 1970

20. Montroll, E.W.: Applied combinatorial mathematics. Beckenbach. F. F. (ed.). New York: Wiley 1964

21. Golden, S.: Lower bounds for the Helmholtz function. Phys. Rev. 137B, 1127-1128 (1965)

Thompson, C.J.: Inequality with applications in statistical mechanics. J. Math. Phys. 6, 1812-1813 (1965)

22. Trotter, H.F.: Approximation of semi-groups of operators. Pacific J. Math. 8, 887-919 (1958) Reed, M., Simon, B.: Methods of modern mathematical physics, Vol. 1. New York: Academic Press 1972

23. Grenander, U., Szegö, G.: Toeplitz forms and their applications. Berkeley, CA: University of California Press 1958

24. Mehta, C.L.: Some inequalities involving traces of operators. J. Math. Phys. 9, 693-697 (1968)

Communicated by J. Fröhlich

Received September 24, 1982; in revised form April 20, 1983

Note added in proof. One of the authors (P.A.P.) has now shown that the mean-field theory for the for the quantum Potts model becomes exact in the $q \rightarrow \infty$ limit. The details will appear elsewhere. 
